\title{
Multiway Filtering Based on Fourth-Order Cumulants
}

\author{
Damien Muti \\ Groupe GSM, Institut Fresnel (UMR CNRS 6133), EGIM, Université Aix-Marseille III, D.U. de Saint Jérôme, \\ 13397 Marseille Cedex 20, France \\ Email:damien.muti@fresnel.fr \\ Salah Bourennane \\ Groupe GSM, Institut Fresnel (UMR CNRS 6133), EGIM, Université Aix-Marseille III, D.U. de Saint Jérôme, \\ 13397 Marseille Cedex 20, France \\ Email: salah.bourennane@fresnel.fr
}

Received 31 March 2004; Revised 4 November 2004; Recommended for Publication by Chong-Yung Chi

\begin{abstract}
We propose a new multiway filtering based on fourth-order cumulants for the denoising of noisy data tensor with correlated Gaussian noise. The classical multiway filtering is based on the TUCKALS3 algorithm that computes a lower-rank tensor approximation. The presented method relies on the statistics of the analyzed multicomponent signal. We first recall how the well-known lower-rank- $\left(K_{1}, \ldots, K_{N}\right)$ tensor approximation processed by TUCKALS3 alternating least square algorithm exploits second-order statistics. Then, we propose to introduce the fourth-order statistics in the TUCKALS3-based method. Indeed, the use of fourthorder cumulants enables to remove the Gaussian components of an additive noise. In the presented method the estimation of the $n$-mode projector on the $n$-mode signal subspace is built from the eigenvectors associated with the largest eigenvalues of a fourthorder cumulant slice matrix instead of a covariance matrix. Each projector is applied by means of the $n$-mode product operator on the $n$-mode of the data tensor. The qualitative results of the improved multiway TUCKALS3-based filterings are shown for the case of noise reduction in a color image and multicomponent seismic data.
\end{abstract}

Keywords and phrases: multicomponent signals, tensors, Tucker3 decomposition, HOSVD, cumulant slice matrix, subspace methods.

\section{INTRODUCTION}

In many fields so diverse as chemometric, psychology, data analysis, or signal processing, a multidimensional and multiway modelling can be adopted in which data are represented by higher-order tensors [1]. A tensor of order $N$ is as a multidimensional array whose entries are accessed via $N$ indices. It is noted $\mathcal{A} \in \mathbb{R}^{I_{1} \times \cdots \times I_{N}}$, where each element is $a_{i_{1} i_{2} \cdots i_{N}}$, and $\mathbb{R}$ is the real manifold. Index $i_{n}$ refers to the $n$-mode of tensor A.

In signal processing, tensors are built on vector spaces associated with physical quantities such as length, width, height, time, color channel, and so forth. Each mode of the tensor is associated with a physical quantity. For example, in image processing, color images can be modelled as thirdorder tensors: two dimensions for lines and columns, and one dimension for the color map. In seismic and underwater acoustics, when a linear antenna is used, a three-dimensional modelling of data can be chosen as well: one mode is associated with the spatial sensors of the antenna, one mode with the time, and one mode with the wave polarization components.
In the classical processing based on algebraic methods, multidimensional and multiway data are split or unfold into observation vectors or matrices in order to apply the classical methods usually based on the covariance matrix. Then, the processed vectors or matrices are merged or rearranged in order to retrieve the size of the initial tensor.

The splitting of multidimensional data may reduce the quantity of information related to the whole tensor as the possibility of studying the relations between components of different slices of data is lost. To overcome this problem, new methods, in which multidimensional data are considered as indivisible whole tensors, have recently been proposed $[2,3,4,5]$. These methods are based on multilinear algebra and on Tucker3 tensor decomposition [6, 7] also known as higher-order SVD (HOSVD) and lower-rank- $\left(K_{1}, \ldots, K_{N}\right)$ tensor approximation $[8,9]$. The Tucker3 decomposition generalizes the matrix singular value decomposition (SVD) [10] to tensors.

Another tensor decomposition model called PARAFAC/ CANDECOMP that performs a canonical decomposition of a tensor into a sum of rank-one tensors has also been proposed by Kruskal [11, 12] and Harshman and Lundy [13]. 
Some PARAFAC/CANDECOMP algorithms have been developed $[12,13,14]$ and applied in food industry [15], array processing [16], and telecommunications [17]. Moreover, several studies on lower-rank-one tensor approximation have been proposed $[9,18,19]$, however, so far, no algorithm is able to perform an orthogonal canonical decomposition of a tensor [20].

The HOSVD mathematical tool has been recently used in image processing for facial expression analysis on data modelled by third-order and fifth-order tensors [21, 22].

In $[3,4]$, a new concept of multiway filtering has been proposed, in which each $n$-mode of an Nth-order data tensor $\mathcal{A}$ is filtered by a matrix $H_{n}$ called $n$-mode filter. Each $n$ mode filter $H_{n}$ is applied to the corresponding $n$-mode vectors of data tensor $\mathcal{A}$ by means of a multilinear algebraic operator called " $n$-mode product" $[8,9]$, and denoted by $\times_{n}$.

In the framework of multidimensional data denoising, two methods have been recently proposed in order to determine the form of $n$-mode filters $H_{n}$. In these two methods, the multidimensional noise is assumed to be additive, white, and Gaussian. In the first method [4], for every $n$ mode, the $n$-mode filter $H_{n}$ is the projector on the $n$-mode signal subspace. These $n$-mode filters are determined thanks to the lower-rank- $\left(K_{1}, \ldots, K_{N}\right)$ noisy data tensor approximation, after convergence of TUCKALS3 algorithm [7, 8, 9]. This method generalizes to tensors the initially developedin-seismic classical matrix filtering [23, 24, 25, 26, 27, 28, 29]. In the second method [3], $n$-mode filters $H_{n}$ are determined by minimizing the mean square error between the estimated signal and the desired signal. This minimization leads to the so-called estimated $n$-mode filters which can be considered as the extension of the well-known Wiener filter in a particular mode.

In both methods the $n$-mode filters can be considered as the projectors on the left singular vectors associated with the largest singular values of the $n$-mode unfolding matrix of the noisy data tensor, or as a weighted version of these projectors.

In this study, we first recall how the singular vectors on which the $n$-mode filters are built can be considered as the eigenvectors associated with the largest eigenvalues of the data $n$-mode vector covariance matrix. Then, we propose a new method to estimate the basis vectors of the $n$ mode signal subspace no more based on second-order moments, but on the noisy data tensor $n$-mode vectors fourthorder cumulants. The goal of this approach is to use the well-known property stating that the higher-order cumulants of a random variable that follows a Gaussian density probability law are null, in order to remove the Gaussian component of the noise. In order to reduce the computational load, and based on the well-known array processing results $[30,31,32]$, in which the determination of the wave directions of arrival (DOA) is estimated from the fourth-order cumulant slice matrix, we also propose to estimate each projector on the $n$-mode signal subspaces thanks to the lower-rank approximation of the fourth-order cumulant slice matrix of the data $n$-mode vectors instead of the fourth-order cumulant tensor lower-rank approximation.
This paper is organized as follows. Section 2 gives the problem statement. Section 3 presents the multiway and multidimensional filtering of a data tensor based on lower-rank- $\left(K_{1}, \ldots, K_{N}\right)$ tensor approximation (LRTA- $\left(K_{1}\right.$, $\left.\ldots, K_{N}\right)$ ). TUCKALS3 alternative least square algorithm that performs LRTA- $\left(K_{1}, \ldots, K_{N}\right)$ is presented and an interpretation in terms of multiway principal component analysis (PCA) is given. In Section 4 a modification of the multiway filtering is proposed by replacing some $n$-mode vector covariance matrix involved in TUCKALS3 algorithm by the fourth-order cumulant slice matrix of these $n$-mode vectors. In Section 5 this new multiway filtering method is applied to noise reduction in a color image and multicomponent seismic data, in the case where the additive Gaussian noise is correlated and independent of the signal.

\section{PROBLEM STATEMENT}

The classical data model is the following. The measurement of a multidimensional and multiway signal $X$ by multicomponent sensors with additive white and Gaussian noise $\mathcal{N}$, statistically independent of the signal, results in data tensor

$$
\mathcal{R}=\mathcal{X}+\mathcal{N} .
$$

Assuming that $\mathcal{X}$ and $\mathcal{N}$ are $N$ th-order tensors from $\mathbb{R}^{I_{1} \times \cdots \times I_{N}}$, the goal of this study is to estimate the desired signal $X$ thanks to a multidimensional filtering of the data $[3,4]$ :

$$
\widehat{X}=\mathcal{R} \times{ }_{1} H^{(1)} \cdots \times_{N} H^{(N)},
$$

where $\times_{n}$ is the $n$-mode product, whose properties can be found in $[8,9]$. The $n$-mode product between a data tensor $\mathcal{R}$ and matrix $H^{(n)}$ represents the consecutive matrix products between matrix $H^{(n)}$ and the $n$-mode vectors of tensor $\mathcal{R}$ defined as the $I_{n}$-dimensional vectors obtained from $\mathcal{R}$ by varying index $i_{n}$ and keeping the other indices fixed. Given $\mathcal{A} \in \mathbb{R}^{I_{1} \times \cdots \times I_{N}}$ and a matrix $U \in \mathbb{R}^{I_{n} \times I_{n}}, \mathscr{B}=\mathcal{A} \times{ }_{n} U$ is a tensor of $\mathbb{R}^{I_{1} \times \cdots \times I_{n-1} \times I_{n} \times I_{n+1} \times \cdots \times I_{N}}$, whose entries are given by

$$
b_{i_{1} \cdots i_{n-1} j_{n} i_{n+1} \cdots i_{N}}=\sum_{i_{n}=1}^{I_{n}} a_{i_{1} \cdots i_{n-1} i_{n} i_{n+1} \cdots i_{N}} u_{j_{n} i_{n}} .
$$

From a signal processing point of view, the $n$-mode product between $\mathcal{R}$ and $H^{(n)}$ is an $n$-mode filtering of data tensor $\mathcal{R}$ by $n$-mode filter $H^{(n)}$. Consequently, for all $n=1$ to $N$, $H^{(n)}$ is the $n$-mode filter applied to the $n$-mode of the data tensor $\mathcal{R}$.

We define $E^{(n)}$ the $n$-mode vector space of dimension $I_{n}$, associated with the $n$-mode of tensor $\mathcal{A}$. By definition, $E^{(n)}$ is generated by the column vectors of the $n$-mode unfolding matrix, where the $n$-mode matrix unfolding $A_{n}$ of tensor $\mathcal{A} \in \mathbb{R}^{I_{1} \times \cdots \times I_{N}}$ is a matrix from $\mathbb{R}^{I_{n} \times M_{n}}$, with

$$
M_{n}=I_{1} \cdots I_{n-1} I_{n+1} \cdots I_{N} .
$$


The columns of $A_{n}$ are the $I_{n}$-dimensional vectors obtained from $\mathcal{A}$ by varying index $i_{n}$ and keeping the other indices fixed, and represent by definition, and in all the following, the $n$-mode vectors of tensor $\mathcal{A}$.

\section{MULTIWAY AND MULTIDIMENSIONAL FILTERING}

In the tensor signal processing assumptions $[2,4,7,9], E^{(n)}$ is the superposition of two orthogonal subspaces:

(i) the signal subspace $E_{1}^{(n)}$ of dimension $K_{n}$,

(ii) and the noise subspace $E_{2}^{(n)}$ of dimension $I_{n}-K_{n}$, such that $E^{(n)}=E_{1}^{(n)} \oplus E_{2}^{(n)}$.

Supposing the dimension $K_{n}$ of the signal subspace is known for all $n=1, \ldots, N$, one way to estimate the desired signal tensor $\mathcal{X}$ from the noisy data tensor $\mathcal{R}=\mathcal{X}+\mathcal{N}$ is to orthogonally project every $n$-mode vector of tensor $\mathcal{R}$ on the $n$-mode signal subspace $E_{1}^{(n)}$, for all $n=1, \ldots, N$. This statement is equivalent to replacing in relation (2) filters $H^{(n)}$ by projectors $P^{(n)}$ on the $n$-mode signal subspace:

$$
\widehat{X}=\mathcal{R} \times{ }_{1} P^{(1)} \cdots \times \times_{N} P^{(N)} .
$$

This multidimensional and multiway filtering generalizes the classical matrix filtering methods $[23,24,25,26,27,28$, 29].

In the one-dimensional case, the definition of the projector on the signal subspace is based on the eigenvectors associated with the largest eigenvalues of the observation vector set covariance matrix [28].

In the multidimensional and multiway case, the projectors on the $n$-mode vector spaces are determined by computing the lower-rank- $\left(K_{1}, \ldots, K_{N}\right)$ tensor approximation (LRTA- $\left.\left(K_{1}, \ldots, K_{N}\right)\right)$ of noisy data tensor $\mathcal{R}[2,4]$, in which $K_{n}$ is the $n$-mode signal subspace dimension for all $n=$ $1, \ldots, N$.

\subsection{Lower-rank- $\left(K_{1}, \ldots, K_{N}\right)$ tensor approximation}

The lower-rank- $\left(K_{1}, \ldots, K_{N}\right)$ approximation of a tensor defined in $[8,9]$ is a concept based on Tucker3 tensor decomposition [6] that generalizes to tensors the singular value decomposition (SVD) [10] and lower-rank approximation of matrices.

The best lower-rank- $\left(K_{1}, \ldots, K_{N}\right)$ approximation $[8,9]$ of a given tensor $\mathcal{R}$, named $\mathcal{B}$, is the tensor of rank$\left(K_{1}, \ldots, K_{N}\right)$ minimizing the Frobenius quadratic distance:

$$
\|\mathcal{R}-\mathscr{B}\|_{F}^{2} \text {. }
$$

The least mean square solution [7] implies that

$$
\mathscr{B}=\mathcal{R} \times{ }_{1} P^{(1)} \cdots \times \times_{N} P^{(N)}
$$

with projectors $P^{(n)}$ defined by $P^{(n)}=U^{(n)} U^{(n)^{T}}$ for all $n=1, \ldots, N$, where the column-wise orthonormal matrices $U^{(n)} \in \mathbb{R}^{K_{n} \times I_{n}}$ maximize the function

$$
g\left(U^{(1)}, \ldots, U^{(N)}\right)=\left\|\mathcal{A} \times_{1} U^{(1)^{T}} \cdots \times_{N} U^{(N)^{T}}\right\|_{F}^{2} .
$$

\subsection{ALS algorithm}

The maximization of function $g$ with respect to $\left(U^{(n)}\right.$, $\left.\ldots, U^{(N)}\right)$, subject to $U^{(n)}$ being a column-wise orthonormal matrix for $n=1, \ldots, N$, is a difficult nonlinear least square problem which is generally solved thanks to TUCKALS3 alternative least square algorithm [7] that can be summarized in the following steps.

(1) Initialization, $k=0$ : for all $n=1, \ldots, N, P_{0}^{(n)}=$ $U_{0}^{(n)} U_{0}^{(n)^{T}} \cdot U_{0}^{(n)}$ is the matrix of the $K_{n}$ left singular vectors associated with the $K_{n}$ largest singular values of tensor $\mathcal{R} n$-mode unfolding matrix $R_{n}$.

(2) ALS loop: repeat until convergence, that is, for example, while $\left\|\mathscr{B}_{k+1}-\mathscr{B}_{k}\right\|_{F}^{2}>\epsilon, \epsilon>0$, prior fixed threshold,

(a) for $n=1$ to $N$,

(i) $\mathscr{B}^{(n), k}=\mathcal{R} \times{ }_{1} P_{k+1}^{(1)} \cdots \times_{n-1} P_{k+1}^{(n-1)} \times_{n+1}$ $P_{k}^{(n+1)} \cdots \times \times_{N} P_{k}^{(N)}$

(ii) $n$-mode unfold tensor $\mathscr{B}^{(n), k}$ into matrix $B_{n}^{(n), k}$

(iii) compute matrix $C^{(n), k}=B_{n}^{(n), k} R_{n}^{T}$;

(iv) process $C^{(n), k}$ eigenvalue decomposition (EVD), put the $K_{n}$ eigenvectors associated with the $K_{n}$ largest eigenvalues into $U_{k+1}^{(n)}$;

(v) compute $P_{k+1}^{(n)}=U_{k+1}^{(n)} U_{k+1}^{(n)^{T}}$;

(b) compute $\mathscr{B}_{k+1}=\mathcal{R} \times{ }_{1} P_{k+1}^{(1)} \cdots \times \times_{N} P_{k+1}^{(N)}, k \leftarrow k+1$.

(3) Output: $\mathscr{B}_{k_{\text {stop }}}=\mathcal{R} \times{ }_{1} P_{k_{\text {stop }}}^{(1)} \cdots \times_{N} P_{k_{\text {stop }}}^{(N)}$, the best lowerrank- $\left(K_{1}, \ldots, K_{N}\right)$ approximation of $\mathcal{R}$, where $k_{\text {stop }}$ is the last iteration after convergence of TUCKALS3 algorithm.

Note that in steps (2)(a)(iii)-(iv) an improvement of TUCKALS3 algorithm which decreases the computational load by using a fast algorithm for the determination of matrix $C^{(n), k}$ eigenvectors has been proposed in $[8,9]$, but will not be discussed in this paper.

\section{3. $\operatorname{LRTA}-\left(K_{1}, \ldots, K_{N}\right)$ and multiway PCA}

In step (2)(a)(i), at TUCKALS3 algorithm $k$ th iteration, and for a given $n$-mode, tensor $\mathcal{B}^{(n), k}$ represents data tensor $\mathcal{R}$ which is filtered in every $m$-mode except in the $n$-mode, by projectors $P_{l}^{(m)}$ in which index $l=k+1$ for $m<n$ and index $l=k$ for $m>n$.

We give a physical sense to matrix $C^{(n), k}$ from step (2)(a)(iii). We define by $\mathbf{b}_{j}^{(n), k}, j=1, \ldots, M_{n}$, with

$$
M_{n}=I_{1}, \ldots, I_{n-1} I_{n+1}, \ldots, I_{N},
$$

the $n$-mode vectors of tensor $\mathscr{B}^{(n), k}$, that is, $n$-mode unfolding matrix $B_{n}^{(n), k}$ column vectors. We define as well by $\mathbf{r}_{j}^{(n)}, j=1, \ldots, M_{n}$, the $n$-mode vectors of tensor $\mathcal{R}$. Matrix $C^{(n), k}=B_{n}^{(n), k} R_{n}^{T}$ can be written as

$$
C^{(n), k}=\left[\mathbf{b}_{1}^{(n), k}, \ldots, \mathbf{b}_{M_{n}}^{(n), k}\right]\left[\mathbf{r}_{1}^{(n)}, \ldots, \mathbf{r}_{M_{n}}^{(n)}\right]^{T}=\sum_{j=1}^{M_{n}} \mathbf{b}_{j}^{(n), k} \mathbf{r}_{j}^{(n)^{T}} .
$$


As a consequence, up to the multiplicative factor $1 / M_{n}$, matrix $C^{(n), k}$ can be considered as an estimation of the covariance matrix between data tensor $\mathcal{R} n$-mode vectors and tensor $\mathscr{B}^{(n), k} n$-mode vectors. Hence, the $K_{n}$ eigenvectors associated with the largest eigenvalues of matrix $C^{(n), k}$ from which projector $P_{k+1}^{(n)}$ is built at step (2)(a)(v) are the mutual principal components of tensor $\mathcal{R}$ and tensor $\mathscr{B}^{(n), k} n$-mode vectors. Thanks to projectors $P_{l}^{(m)}, l=k+1$ for $m<n$ and $l=k$ for $m>n$, from the expression of tensor $\mathscr{B}^{(n), k}$ (step (2) (a)(i)), these principal components in the $n$-mode depend on the principal components in the $m$-modes, for $m \neq n$.

Thus the lower-rank- $\left(K_{1}, \ldots, K_{N}\right)$ tensor approximation of data tensor $\mathcal{R}$ consists of a principal component analysis processed alternatingly on every $n$-mode, where all the $n$-mode principal components are interdependent, and are supposed to define the signal subspace entirely.

Consequently, in the context of signal processing, the TUCKALS3 algorithm gradually refines, with respect to iteration $k$, the estimation of orthogonal projectors $P_{k+1}^{(n)}$ on associated $n$-mode signal subspace, for all $n=1, \ldots, N$. In the next sections the goal of our study is to improve the estimation of these orthogonal projectors on the $n$-mode signal subspaces. A proposition of improvement based on higherorder statistics, in the case of an additive correlated Gaussian noise, is presented.

\section{MULTIWAY FILTERING USING THE FOURTH-ORDER CUMULANTS}

\subsection{Second-order moments in TUCKALS3 algorithm}

In previous Section 3.3, it is shown that, up to the multiplicative factor $1 / M_{n}$, matrix $C^{(n), k}$ is an estimation of the covariance matrix between data tensor $\mathcal{R} n$-mode vectors and tensor $\mathcal{B}^{(n), k} n$-mode vectors. Considering, in relation (10), that $\left\{\mathbf{r}_{j}^{(n)}, j=1, \ldots, M_{n}\right\}$ and $\left\{\mathbf{b}_{j}^{(n), k}, j=1, \ldots, M_{n}\right\}$ are the $M_{n}$ realizations of two random vectors $\mathbf{r}^{(n)}$ and $\mathbf{b}^{(n), k}$ associated, respectively, with the $n$-mode vectors of data tensors $\mathcal{R}$ and $\mathscr{B}^{(n), k}$, matrix $C^{(n), k}$ can be written as a second-order moment:

$$
C^{(n), k}=\mathrm{E}\left[\mathbf{b}^{(n), k} \mathbf{r}^{(n)^{T}}\right]
$$

where $\mathrm{E}[\cdot]$ denotes the expectation operator.

Thus, in TUCKALS3 $k$ th iteration, orthogonal projector $P_{k+1}^{(n)}$ on the $n$-mode signal subspace is estimated from a second-order moment since it is built from matrix $C^{(n), k}$ eigenvalue decomposition (EVD).

\subsection{Proposed TUCKALS3 algorithm based on fourth-order cumulants}

In practice, the noise whiteness is not always fulfilled. In the case of additive correlated Gaussian noise, the estimation of orthogonal projector $P_{k+1}^{(n)}$ on the $n$-mode signal subspace cannot be achieved correctly with the classical TUCKALS3 algorithm since it is based on the second-order moments.
One way to improve the estimation of the orthogonal projector $P_{k+1}^{(n)}$ on the $n$-mode signal subspace in the case of additive correlated Gaussian noise is to replace secondorder moment matrix $C^{(n), k}$ with higher-order cumulants. Indeed, the use of higher-order statistics consists of a classical means to remove the noise Gaussian components since the higher-order cumulants of a Gaussian random variable are null $[33,34]$.

Then, the goal of the proposed method is to estimate orthogonal projector $P_{k+1}^{(n)}$ on the $n$-mode signal subspace no more with second-order moment matrix $C^{(n), k}$, but with the higher-order cumulants of the associated $n$-mode vectors.

\subsubsection{Fourth-order cumulants}

As noticed in Section 4.1, matrix $C^{(n), k}$, at step (2)(a)(iii) and TUCKALS3 algorithm $k$ th iteration, is defined as a secondorder moment. In order to remove the noise Gaussian component, we propose to use the fourth-order cumulant tensor

$$
\mathbf{C}^{(n), k}=\operatorname{Cum}\left(\mathbf{b}^{(n), k}, \mathbf{b}^{(n), k^{T}}, \mathbf{r}^{(n)}, \mathbf{r}^{(n)^{T}}\right),
$$

where $\operatorname{Cum}(\cdot)$ denotes the cumulant operator. $\mathbf{C}^{(n), k}$ is a fourth-order supersymmetric tensor from $\mathbb{R}^{I_{n} \times I_{n} \times I_{n} \times I_{n}}$, whose generic term at position $\left(i_{1}, i_{2}, j_{1}, j_{2}\right)$ is given by

$$
\mathbf{C}_{i_{1}, i_{2}, j_{1}, j_{2}}^{(n), k}=\operatorname{Cum}\left(b_{i_{1}}^{(n), k}, b_{i_{2}}^{(n), k}, r_{j_{1}}^{(n)}, r_{j_{2}}^{(n)}\right),
$$

where $b_{i}^{(n), k}$ and $r_{j}^{(n)}$ are the $i$ th and $j$ th components of random vectors $\mathbf{b}^{(n), k}$ and $\mathbf{r}^{(n)}$ associated, respectively, to $n$-mode vectors of tensor $\mathscr{B}^{(n), k}$ and $\mathcal{R}$. According to $[33,35]$, the generic term of $\mathbf{C}^{(n), k}$ can be expressed, thanks to the expectation operator, and for centered random variables, as

$$
\begin{aligned}
\mathrm{C}_{i_{1}, i_{2}, j_{1}, j_{2}}^{(n), k}= & \mathrm{E}\left[b_{i_{1}}^{(n), k} b_{i_{2}}^{(n), k} r_{j_{1}}^{(n)} r_{j_{2}}^{(n)}\right] \\
& -\mathrm{E}\left[b_{i_{1}}^{(n), k} r_{j_{1}}^{(n)}\right] \mathrm{E}\left[b_{i_{2}}^{(n), k} r_{j_{2}}^{(n)}\right] \\
& -\mathrm{E}\left[b_{i_{1}}^{(n), k} r_{j_{2}}^{(n)}\right] \mathrm{E}\left[b_{i_{2}}^{(n), k} r_{j_{1}}^{(n)}\right] .
\end{aligned}
$$

At step (2)(a)(iv), the determination of the $K_{n}$ eigenvectors associated with the $K_{n}$ largest eigenvalues of covariance matrix $C^{(n), k}$ amounts to compute its best lower-rank- $K_{n}$ approximation according to Eckart-Young theorem [36]. One way to generalize matrix $C^{(n), k}$ lower-rank approximation to fourth-order cumulant tensor $\mathbf{C}^{(n), k}$ is to compute $\mathbf{C}^{(n), k}$ lower-rank- $\left(K_{n}, K_{n}, K_{n}, K_{n}\right)$ tensor approximation, thanks to TUCKALS3 algorithm $[8,37]$. Using the fact that $\mathbf{C}^{(n), k}$ is a supersymmetric tensor, we get $[8,37]$

$$
\mathbf{C}^{(n), k}=\mathbf{S}_{K_{n}}^{(n), k} \times_{1} U_{k+1}^{(n)} \times_{2} U_{k+1}^{(n)} \times_{3} U_{k+1}^{(n)} \times_{4} U_{k+1}^{(n)},
$$

in which $\mathbf{S}_{K_{n}}^{(n), k} \in \mathbb{R}^{K_{n} \times K_{n} \times K_{n} \times K_{n}}$ is Tucker3 core tensor [7], and matrix $U_{k+1}^{(n)} \in \mathbb{R}^{I_{n} \times K_{n}}$ is a column-wise orthogonal matrix.

A good approximation of matrix $U_{k+1}^{(n)}$ involved in $\mathrm{C}^{(n), k}$ lower-rank- $\left(K_{n}, K_{n}, K_{n}, K_{n}\right)$ tensor approximation consists in keeping the $K_{n}$ first orthogonal vectors of unitary 
matrix $V_{k+1}^{(n)}$ involved in tensor $\mathbf{C}^{(n), k}$ HOSVD, in which matrix $V_{k+1}^{(n)}$ satisfies relation (15) in the particular case when $K_{n}=I_{n}$. This process is usually called rank$\left(K_{n}, K_{n}, K_{n}, K_{n}\right)$ HOSVD truncation of tensor $\mathbf{C}^{(n), k}$. The rank- $\left(K_{n}, K_{n}, K_{n}, K_{n}\right)$ HOSVD truncation of tensor $\mathbf{C}^{(n), k}$ is not the best lower-rank- $\left(K_{n}, K_{n}, K_{n}, K_{n}\right)$ tensor approximation of $\mathbf{C}^{(n), k}[8,9]$. Nevertheless, according to $[8,9]$ and confirmed by several experimental simulations [5], the error done at the minimization of $\left\|\mathbf{C}^{(n), k}-\mathbf{B}\right\|_{F}^{2}$, with $\mathbf{B}$ being a rank- $\left(K_{n}, K_{n}, K_{n}, K_{n}\right)$ tensor (see relation (6)), is low compared to the gain in computational time earned by avoiding the ALS loop of TUCKALS3 algorithm.

However, the main drawback of this method is the high computational time to build every fourth-order cumulant tensor associated to the $n$-mode of the data tensor. This computational time depends on the size of data tensor $\mathcal{R}$, that is, the values of $I_{n}$, for all $n=1 \ldots, N$. One way to reduce the computational load is to use the fourth-order cumulant slice matrix which is extracted from the fourth-order cumulant tensor. This is done in the following subsection.

\subsubsection{Fourth-order cumulant slice matrix}

In many applications of array processing $[32,38]$, the fourthorder cumulant matrix consisting of all possible permutations of the four indices $\left\{i_{1}, i_{2}, j_{1}, j_{2}\right\}$ is used rather than fourth-order cumulant tensor $\mathbf{C}^{(n), k}$. The cumulant matrix is a matrix representation of the cumulant tensor. In order to reduce the computational time necessary to build and analyse the cumulant matrix, a cumulant slice matrix of $\mathbf{C}^{(n), k}$ has been introduced in $[30,31,32]$.

The cumulant slice matrix associated with the first component of vector $\mathbf{b}^{(n), k}$ is given by the following $\left(I_{n} \times I_{n}\right)$ Hermitian matrix $[30,31,32]$ :

$$
\mathbf{C}_{1}^{(n), k}=\operatorname{Cum}\left(b_{1}^{(n), k}, b_{1}^{(n), k}, \mathbf{r}^{(n)}, \mathbf{r}^{(n)^{T}}\right) .
$$

The generic $(i, j)$-term of cumulant slice $\mathbf{C}_{1}^{(n), k}$ expressed with the expectation operator is

$$
\mathrm{C}_{1 i j}^{(n), k}=\mathrm{E}\left[\left(b_{1}^{(n), k}\right)^{2} r_{i}^{(n)} r_{j}^{(n)}\right]-2 \mathrm{E}\left[b_{1}^{(n), k} r_{i}^{(n)}\right] \mathrm{E}\left[b_{1}^{(n), k} r_{j}^{(n)}\right] .
$$

The practical estimation of $\mathbf{C}_{1}^{(n), k}$ uses the $M_{n}$ realizations of random vectors $\mathbf{r}^{(n)}$ and $\mathbf{b}^{(n), k}$, where $M_{n}$ is defined in relation (9). Defining by $b_{i j}^{(n), k}$ and $r_{i j}^{(n)}$ the $(i, j)$-term of $n$-mode unfolding matrices $B_{n}^{(n), k}$ and $R_{n}$, the estimation of $\mathrm{C}_{1 i j}^{(n), k}(i, j)$-term is given by

$$
\begin{aligned}
\mathrm{C}_{1 i j}^{(n), k}= & \frac{1}{M_{n}}\left(\sum_{p=1}^{M_{n}}\left(b_{1 p}^{(n), k}\right)^{2} r_{i p}^{(n)} r_{j p}^{(n)}\right) \\
& -\frac{2}{M_{n}^{2}}\left(\sum_{p=1}^{M_{n}} b_{1 p}^{(n), k} r_{i p}^{(n)}\right)\left(\sum_{p=1}^{M_{n}} b_{1 p}^{(n), k} r_{j p}^{(n)}\right) .
\end{aligned}
$$

Relations (17) and (18) can be adapted to noncentered random variables $[33,35]$.
Initially, the use of the fourth-order cumulant slice matrix has been proposed in the array processing field for locating sources $[30,31,32]$. The results obtained in array processing state that the use of the fourth-order cumulant slice matrix, instead of the cumulant matrix, gives a good compromise between the localisation performances and the computational load [38].

In this paper, our aim is to improve the LRTA$\left(K_{1}, \ldots, K_{N}\right)$-based multiway filtering of multicomponent seismic data and color images, in the case of additive correlated Gaussian noise. As shown in Figures 1 and 2, the performances of the improved fourth-order-cumulantbased LRTA- $\left(K_{1}, \ldots, K_{N}\right)$ filtering involving, respectively, the fourth-order cumulant tensor and the fourth-order cumulant slice matrix are sensibly identical. Thus, according to the results obtained in array processing $[30,31,32]$, and according to our simulations on multicomponent seismic signal and color image filtering, we have used the cumulant slice matrix in order to reduce the computational load of the improved fourth-order cumulant-LRTA- $\left(K_{1}, \ldots, K_{N}\right)$-based filtering.

The gain in computational load brought by the use of the fourth-order cumulant slice matrix rather than the cumulant tensor can be explained by at least two reasons.

(1) Firstly, as the fourth-order cumulant tensor in the $n$ mode consists of $I_{n}^{4}$ elements, the number of operations required for its construction is squared compared to the one required for the construction of the cumulant slice matrix which only consists of $I_{n}^{2}$ elements. Moreover, depending of the data size, the memory space occupied by the fourth-order cumulant tensor can become too important for usual computers.

(2) Secondly, the estimation of the projector on the $n$ mode subspace requires only one EVD when the cumulant slice matrix is used where as it requires 4 SVDs multiplied by the number of iterations necessary for the convergence of TUCKALS3 algorithm involved in the fourth-order cumulant tensor lowerrank- $\left(K_{n}, K_{n}, K_{n}, K_{n}\right)$ tensor approximation. We note that the determination of the eigenvectors (or singular vectors) associated with the largest eigenvalues (or singular values), involved in the construction of the projector on the $n$-mode subspace, can be accelerated, thanks to fast algorithms such as the power method [10] (for the cumulant slice matrix) or the higherorder power method $[8,9]$ (for the cumulant tensor).

Hence, in the following section, in regard to the filtering simulations treated in this paper, we consider that the eigenvectors associated with the largest non-zero eigenvalues of fourth-order cumulant slice matrix $\mathbf{C}_{1}^{(n), k}$ consist of a good approximation of the $n$-mode signal subspace basis since the fourth-order cumulant tensor of noise $\mathcal{N} n$-mode vectors is null and since the probability density function of random vector $\mathbf{x}^{(n)}$ associated with signal tensor $\mathcal{X} n$-mode vectors is not Gaussian.

In the following subsection, the proposed algorithm is summarized. 


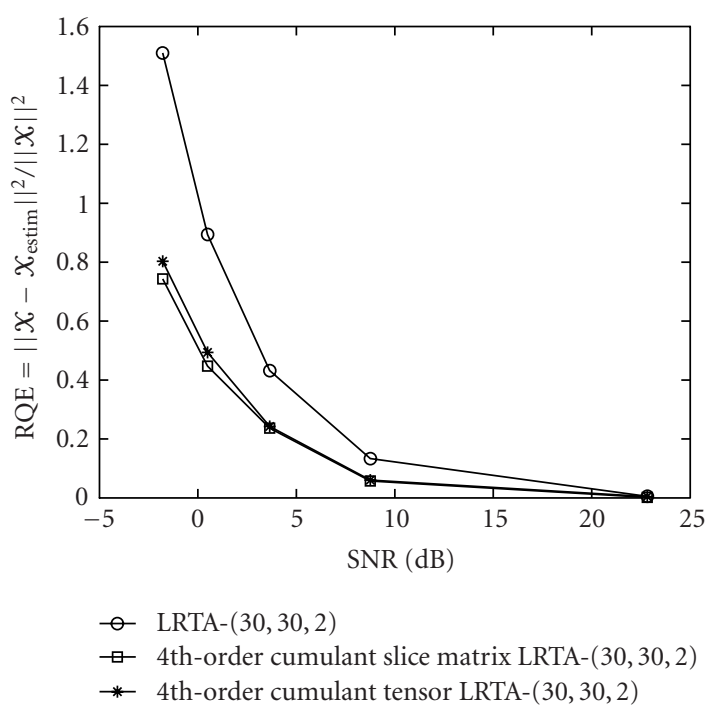

FIgURE 1: RQE evolution with respect to SNR (dB) for LRTA$(30,30,2)$-based (square), fourth-order-cumulant-slice-matrix LRTA-(30, 30, 2)-based (circle), and fourth-order-cumulant-tensor LRTA-(30,30,2)-based (asterisk) multiway filterings of Baboon standard image.

\subsubsection{Proposed algorithm}

The proposed method is based on the classical multiway filtering method. In step (2)(a)(ii), and iteration $k$ of TUCKALS3 algorithm involved in data tensor $\mathcal{R}$ lower-rank$\left(K_{1}, \ldots, K_{N}\right)$ tensor approximation, and as far as the $n$-mode is concerned, matrix $C^{(n), k}$ representing the covariance matrix between data tensor $\mathcal{R}$ and tensor $\mathscr{B}^{(n), k} n$-mode vectors is replaced by the fourth-order cumulant slice matrix $\mathbf{C}_{1}^{(n), k}$ of the same $n$-mode vectors. $\mathbf{C}_{\mathbf{1}}^{(n), k}$ is built by using, for example, the first component of $\mathbf{b}^{(n), k}$ random vectors as the reference element associated with the fourth-order cumulant slice matrix. The practical estimation of its generic term is given in relation (18). Then, in step (2)(a)(v), projector $P_{k+1}^{(n)}$ represents the projection on the $K_{n}$ eigenvectors associated with the $K_{n}$ largest eigenvalues of fourth-order cumulant slice ma$\operatorname{trix} \mathbf{C}_{1}^{(n), k}$.

TUCKALS3 algorithm initialization step is also modified by using the fourth-order cumulant slice matrix. For $n=1, \ldots, N$, matrix $U_{0}^{(n)}$, with which initial projector $P_{0}^{(n)}$ is built, is the matrix of the $K_{n}$ left singular vectors associated with the $K_{n}$ largest singular values of $n$-mode unfolding matrix $R_{n}$. By definition, $U_{0}^{(n)}$ is the matrix of the $K_{n}$ eigenvectors associated with Hermitian matrix $R_{n} R_{n}^{T}$, which, according to the remarks made on relation (10), consists of an estimation of data tensor $\mathcal{R} n$-mode vector covariance matrix. In the proposed method, in the initialization step, matrix $R_{n} R_{n}^{T}$ is replaced with $\mathrm{C}_{1}^{(n), 0}$, the fourthorder cumulant slice matrix of data tensor $\mathcal{R} n$-mode vectors, which is estimated, thanks to relation (18), by replacing the element $b_{1 p}^{(n), k}$ by the element $r_{1 p}^{(n)}$ for all $p=$ $1, \ldots, M_{n}$.

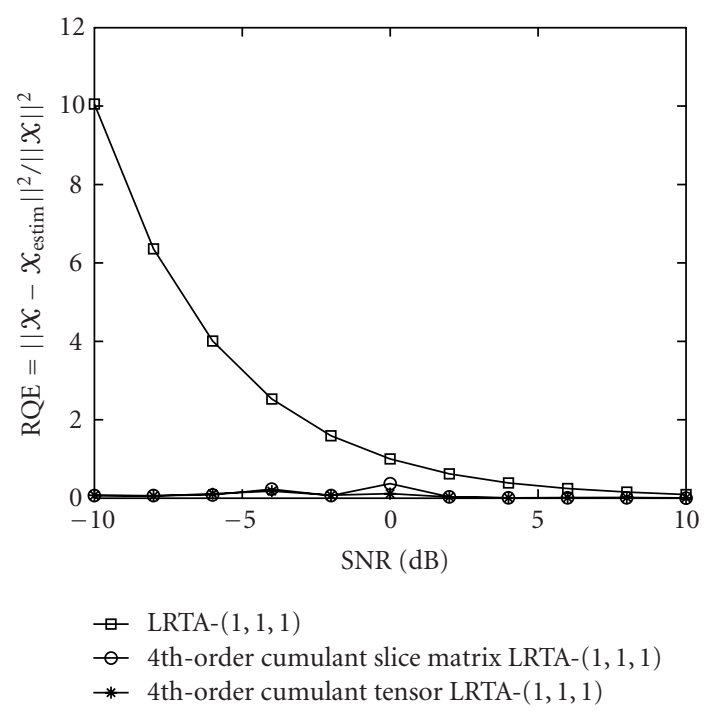

FIGURe 2: RQE evolution with respect to SNR (dB) for classical LRTA-(1, 1, 1)-based (square), fourth-order-cumulant-slice-LRTA$(1,1,1)$-based (circle), and fourth-order-cumulant-tensor-LRTA$(1,1,1)$-based (asterisk) multiway filterings applied on a multicomponent seismic wave with totally correlated Gaussian noise (Figure 5).

The improved fourth-order cumulant-slice-matrixLRTA- $\left(K_{1}, \ldots, K_{N}\right)$-based multiway filtering, for which the first component of every random vector has been chosen as the reference element for the fourth-order cumulant slice matrix computation, can be summarized as follows.

(1) Initialization, $k=0$ : for all $n=1, \ldots, N, P_{0}^{(n)}=$ $U_{0}^{(n)} U_{0}^{(n)^{T}} \cdot U_{0}^{(n)}$ is the matrix of the $K_{n}$ eigenvectors associated with the $K_{n}$ largest eigenvalues of fourthorder cumulant slice matrix $\mathbf{C}_{\mathbf{1}}^{(n), 0}$ of tensor $\mathcal{R} n$-mode vectors.

(2) ALS loop: repeat until convergence, that is, for example, while $\left\|\mathscr{B}_{k+1}-\mathscr{B}_{k}\right\|_{F}^{2}>\epsilon, \epsilon>0$, prior fixed threshold,

(a) for $n=1$ to $N$,

(i) $\mathscr{B}^{(n), k}=\mathcal{R} \times_{1} P_{k+1}^{(1)} \cdots \times_{n-1} P_{k+1}^{(n-1)} \times_{n+1}$ $P_{k}^{(n+1)} \cdots \times_{N} P_{k}^{(N)}$;

(ii) compute cumulant slice $\mathbf{C}_{1}^{(n), k}$ associated with the fourth-order cumulant of tensor $\mathcal{R}$ and tensor $\mathscr{B}^{(n), k} n$-mode vectors. Every element of $\mathbf{C}_{1}^{(n), k}$ is given by relation (18);

(iii) process matrix $\mathbf{C}_{1}^{(n), k}$ eigenvalue decomposition (EVD) and put the $K_{n}$ eigenvectors associated with the $K_{n}$ largest eigenvalues into $U_{k+1}^{(n)}$;

(iv) compute $P_{k+1}^{(n)}=U_{k+1}^{(n)} U_{k+1}^{(n)^{T}}$;

(b) compute $\mathcal{B}_{k+1}=\mathcal{R} \times{ }_{1} P_{k+1}^{(1)} \cdots \times_{N} P_{k+1}^{(N)}, k \leftarrow k+1$.

(3) Output: $\widehat{X}=\mathscr{B}_{k_{\text {stop }}}=\mathcal{R} \times_{1} P_{k_{\text {stop }}}^{(1)} \cdots \times_{N} P_{k_{\text {stop }}}^{(N)}$.

In the next section, this algorithm is used to estimate signal tensor $\widehat{x}$ from noisy data tensor $\mathcal{R}$ representing multicomponent seismic waves, and color images. 


\section{SIMULATION RESULTS}

In the following simulations, the fourth-order-cumulantslice-matrix-lower-rank- $\left(K_{1}, \ldots, K_{N}\right)$-tensor-approximationbased and the classical lower-rank- $\left(K_{1}, \ldots, K_{N}\right)$ tensorapproximation-based multiway filterings are applied to noise reduction in a color image and a multicomponent seismic wave, both of which can be modelled by a third-order tensor from $\mathbb{R}^{I_{1} \times I_{2} \times I_{3}}$. In both applications, the efficiency of the denoising is tested in the presence of additive correlated Gaussian noise.

\subsection{A quality criterion}

According to the previous assumptions, the multidimensional and multicomponent noisy data tensor can be modelled by $\mathcal{R}=\mathcal{X}+\mathcal{N}$, in which $X$ is the signal tensor to estimate and $\mathcal{N}$ is the additive noise tensor. Using the tensor Frobenius norm, we define the signal-to-noise ratio (SNR in $\mathrm{dB}$ ) in the noisy data tensor by

$$
\mathrm{SNR}=10 \log \left(\frac{\|\mathcal{X}\|_{F}^{2}}{\|\mathcal{N}\|_{F}^{2}}\right)
$$

The estimated signal tensor is given by

$$
\widehat{x}=\mathcal{R} \times{ }_{1} P^{(1)} \times_{2} P^{(2)} \times_{3} P^{(3)},
$$

in which $P^{(n)}, n \in\{1,2,3\}$, is the estimated projector on the $n$-mode signal subspace after convergence of the classical LRTA- $\left(K_{1}, K_{2}, K_{3}\right)$-based or the fourth-order-cumulant-slice matrix-LRTA- $\left(K_{1}, K_{2}, K_{3}\right)$-based algorithm.

In order to a posteriori verify the estimated signal tensor quality we propose to use the relative quadratic error criterion $(\mathrm{RQE})$ defined by

$$
\operatorname{RQE}(\widehat{x})=\frac{\|\widehat{x}-X\|_{F}^{2}}{\|X\|_{F}^{2}}
$$

The RQE criterion enables a qualitative comparison between the multiway filtering using the lower-rank- $\left(K_{1}, K_{2}, K_{3}\right)$ tensor approximation and the proposed fourth-ordercumulant-slice-matrix-LRTA- $\left(K_{1}, K_{2}, K_{3}\right)$-based method. Considering this criterion, we expect the fourth-ordercumulant-slice-matrix-LRTA- $\left(K_{1}, K_{2}, K_{3}\right)$-based method to give better (or at least the same) results than the classical LRTA- $\left(K_{1}, K_{2}, K_{3}\right)$-based multiway and multidimensional filtering.

\subsection{Simulations involving correlated Gaussian noise}

A multidimensional and multiway white Gaussian noise $\mathcal{N}$ can be modelled by

$$
\mathcal{N}=\alpha \cdot g,
$$

in which every element of $q \in \mathbb{R}^{I_{1} \times I_{2} \times I_{3}}$ is an independent realization of a normalized centered Gaussian law, and where $\alpha$ is a coefficient that enables to set the SNR in noisy data tensor $\mathcal{R}$.

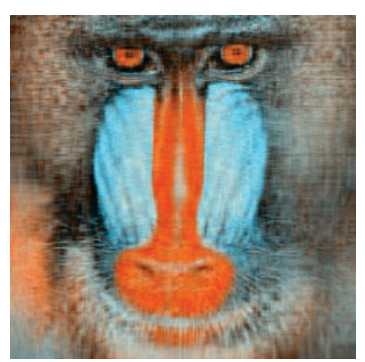

(a)

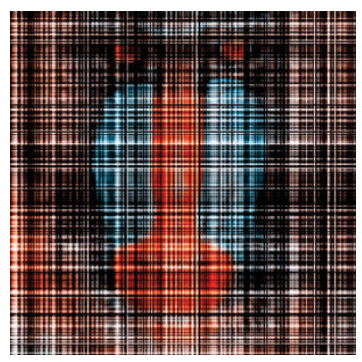

(c)

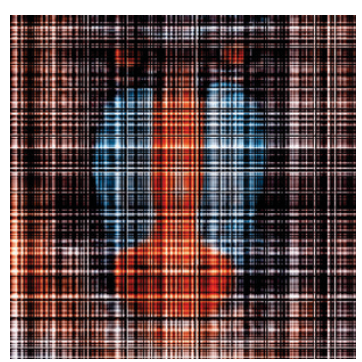

(b)

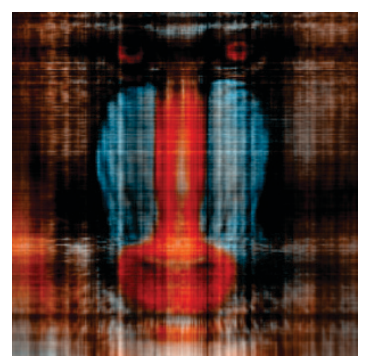

(d)
Figure 3: (a) "Baboon" standard $(256 \times 256 \times 3)$ image. (b) Image with additive correlated Gaussian noise (SNR $=-2.1 \mathrm{~dB}$ ). (c) Classical LRTA- $(30,30,2)$-based multiway filtering of noisy image (b). (d) Fourth-order-cumulant-slice-matrix-LRTA-(30, 30, 2)based multiway filtering of noisy image (b).

A correlated Gaussian noise, named $\mathcal{N}^{c}$, can be built from previous Gaussian noise $\mathcal{N} \in \mathbb{R}^{I_{1} \times I_{2} \times I_{3}}$ given in relation (22) by applying on every $n$-mode of tensor $\mathcal{N}$ a weighting matrix $W^{(n)}$, for all $n=1,2,3$ :

$$
\mathcal{N}^{c}=\mathcal{N} \times_{1} W^{(1)} \times_{2} W^{(2)} \times_{3} W^{(3)} .
$$

Each element of tensor $\mathcal{N}^{c}$, given by

$$
n_{i_{1} i_{2} i_{3}}^{c}=\sum_{k_{1}=1}^{I_{1}} \sum_{k_{3}=1}^{I_{3}} \sum_{k_{3}=1}^{I_{3}} n_{i_{1} i_{2} i_{3}} w_{i_{1} k_{1}}^{(1)} w_{i_{2} k_{2}}^{(2)} w_{i_{3} k_{3}}^{(3)},
$$

is a linear mapping of every element of tensor $\mathcal{N}$, which by definition creates the correlation between every $n$-mode of noise $\mathcal{N}^{c}$.

In the following subsections, classical LRTA- $\left(K_{1}, K_{2}, K_{3}\right)$ filtering and fourth-order-cumulant-slice-matrix-LRTA$\left(K_{1}, K_{2}, K_{3}\right)$-based filtering are applied to a color image and a multicomponent seismic wave.

\subsubsection{Color images}

We consider Baboon standard color image represented on Figure 3a, and modelled by third-order tensor $\mathcal{X} \in$ $\mathbb{R}^{256 \times 256 \times 3}$. Some signal-independent correlated Gaussian noise $\mathcal{N}^{c}$ defined by relation (23) is added to tensor $\mathcal{X}$ and results in noisy image $\mathcal{R}=\mathcal{X}+\mathcal{N}^{c}$ represented on Figure $3 \mathrm{~b}$. In this simulation, coefficient $\alpha$ in relation (22) and the elements of weighting matrix $W^{(n)}$ in relation (23), for all 
$n=1,2,3$, are chosen such that the SNR in noisy image represented on Figure $3 \mathrm{~b}$ is $9 \mathrm{~dB}$, and such that in every $n$-mode, noise tensor $\mathcal{N}^{c} n$-mode vectors are totally correlated.

In these simulations, parameters $\left(K_{1}, K_{2}, K_{3}\right)$ of lowerrank- $\left(K_{1}, K_{2}, K_{3}\right)$ tensor approximation are supposed known and fixed at

$$
\left(K_{1}, K_{2}, K_{3}\right)=(30,30,2) .
$$

Experimentally, these parameters can be chosen from parameters $\left(Q_{1}, Q_{2}, Q_{3}\right)$ that enable lower-rank- $\left(Q_{1}, Q_{2}, Q_{3}\right)$ tensor approximation of initial non noisy color image $X$ to reconstruct a visually non deformed image. This latter image approximation can be considered as a compression with loss of color image $\mathcal{X}$, thus some compression quality criterion can be used to determine some suitable $\left(Q_{1}, Q_{2}, Q_{3}\right)$ parameters, but will not be discussed in this paper.

The LRTA- $(30,30,2)$ filtering of noisy image $\mathcal{R}$ yields to the image of Figure $3 \mathrm{c}$ and the fourth-order-cumulant-slicematrix-LRTA-(30,30,2)-based multiway filtering of noisy image $\mathcal{R}$ yields to the image of Figure $3 \mathrm{~d}$.

The RQE, defined in relation (21), enables a qualitative comparison between the lower-rank- $(30,30,2)$-tensorapproximation-based multiway filtering and the proposed method. Figure 1, that represents the evolution of the $\mathrm{RQE}$ with respect to the SNR varying from $-3 \mathrm{~dB}$ to $23 \mathrm{~dB}$, shows that the proposed fourth-order-cumulantslice-matrix-LRTA- $(30,30,2)$-based (square) and the fourthorder-cumulant-tensor-LRTA-(30, 30, 2)-based (stars) multiway filtering method give sensibly the same results.

However, the principal outcome of this figure is that the proposed fourth-order-cumulant-slice-matrix-LRTA$(30,30,2)$-based multiway filtering gives better results than the classical LRTA-(30,30,2)-based multiway filtering method since, all over the SNR range, the RQE given by the proposed method is lower than the one given by the classical method. This result is confirmed by the good quality of the restored image in Figure 3d compared to the restored image in Figure 3c.

In conclusion, for this simulation, the fourth-ordercumulant-slice-matrix-LRTA-(30, 30, 2)-based multiway filtering gives better results than the classical LRTA- $(30,30,2)$ based multiway filtering with regard to tensor estimation quality and considering the RQE criterion, in the case of additive and signal-independent correlated Gaussian noise.

\subsubsection{Multicomponent seismic signals}

In this simulation, a multicomponent seismic wave is received on a linear antenna composed of 10 sensors. The wave direction of propagation is supposed to be orthogonal to the antenna plane. The three components of the signal wave are called channel 1, channel 2, and channel 3 and are represented on Figures 4a, 4b, 4c, and on Figures 5a, 5b, 5c. In each seismic slice, the $x$-axis corresponds to the time sampling (200 time samples) and the $y$-axis to the spatial sensors (10 sensors). Each component is represented by a triangular impulse with the same temporal length but different amplitude.
Example 1. The multidimensional and multicomponent seismic record is modelled by noisy data tensor $\mathcal{R} \in$ $\mathbb{R}^{10 \times 200 \times 3}$ which is the sum between multicomponent seismic signal $\mathcal{X}$ that we want to estimate and correlated Gaussian noise $\mathcal{N}^{c}$ defined by relation (23). The three components of noisy signal $\mathcal{R}$ are represented, respectively, on Figures $4 \mathrm{~d}$, $4 \mathrm{e}$, and $4 \mathrm{f}$, in which coefficient $\alpha$ in relation (22) and the elements of weighting matrix $W^{(n)}$ in relation (23), for all $n=1,2,3$, are chosen such that $\mathrm{SNR}=-2 \mathrm{~dB}$.

Moreover, in this simulation, each weighting matrix $W^{(n)}, n \in\{1,2,3\}$, from relation (23), is chosen as being a random matrix whose elements are the realizations of a normalized centered Gaussian density probability law.

Parameters $\left(K_{1}, K_{2}, K_{3}\right)$ of lower-rank- $\left(K_{1}, K_{2}, K_{3}\right)$ tensor approximation are supposed to be known and fixed at $(1,1,1)$. The classical LRTA- $(1,1,1)$-based multiway filtering of $\mathcal{R}$ results in estimated signal $\widehat{X}_{1}$ whose three components are represented, respectively, on Figures $4 \mathrm{~g}$, $4 \mathrm{~h}$, and $4 \mathrm{i}$.

The fourth-order-cumulant-slice-matrix-LRTA- $(1,1,1)$ based multiway filtering of data tensor $\mathcal{R}$ results in estimated signal $\widehat{X_{2}}$ whose three components are represented, respectively, on Figures $4 \mathrm{j}, 4 \mathrm{k}$, and $4 \mathrm{l}$.

Finally, the evolution of the RQE with respect to the SNR, represented on Figure 6, shows that for the range of SNR going from $-2 \mathrm{~dB}$ to $5 \mathrm{~dB}$, fourth-order-cumulant-slicematrix-LRTA- $(1,1,1)$-based multiway filtering gives better results than the classical LRTA- $(1,1,1)$-based multiway filtering since, all over the SNR range, the RQE given by the first method is lower than the one given by the second method.

One can also note that in Figure 4, for an SNR = $-2 \mathrm{~dB}$, the signals corresponding to the first two channels of the multicomponent seismic wave, estimated by the classical LRTA- $(1,1,1)$-based multiway filtering, are distorted compared to the ones estimated by the fourth-ordercumulant-slice-LRTA- $(1,1,1)$-based multiway filtering. In addition, the signal amplitude of each channel is well estimated with the fourth-order-cumulant-slice-matrix-LRTA$(1,1,1)$-based multiway filtering, whereas it is not with the classical LRTA- $(1,1,1)$-based multiway filtering.

Example 2. In this simulation, we consider that the noise tensor $\mathcal{N}^{c} 2$-mode vectors are totally correlated. We recall that the 2-mode index of tensor $\mathcal{R} \in \mathbb{R}^{10 \times 200 \times 3}$ is associated to the time quantity; thus, a given 2 -mode vector represents the temporal signal received at a given spatial sensor in a given component.

The three components of noisy signal $\mathcal{R}$ are represented, respectively, in Figures 5d, 5e, and 5f, in which coefficient $\alpha$ in relation (22) and the elements of weighting matrix $W^{(n)}$ in relation (23), for all $n=1,2,3$, are chosen such that SNR $=$ $-10 \mathrm{~dB}$.

Like in Example 1, parameters $\left(K_{1}, K_{2}, K_{3}\right)$ of lowerrank- $\left(K_{1}, K_{2}, K_{3}\right)$ tensor approximation are supposed known and fixed at $(1,1,1)$. The LRTA- $(1,1,1)$ filtering of $\mathcal{R}$ results in estimated signal $\widehat{X_{1}}$ whose three components are represented, respectively, on Figures 5g, 5h, and 5i. According to these figures, the classical method is incapable of determining the $n$-mode signal subspace with this type of noise. 


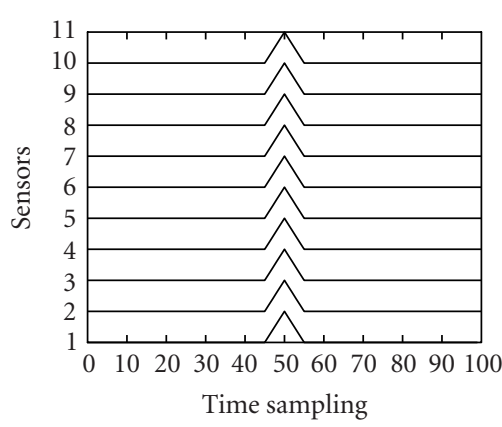

(a)

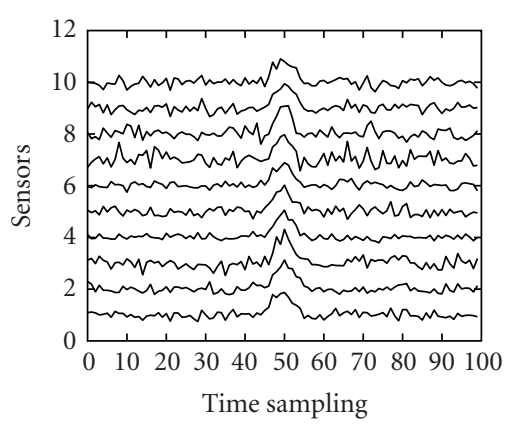

(d)

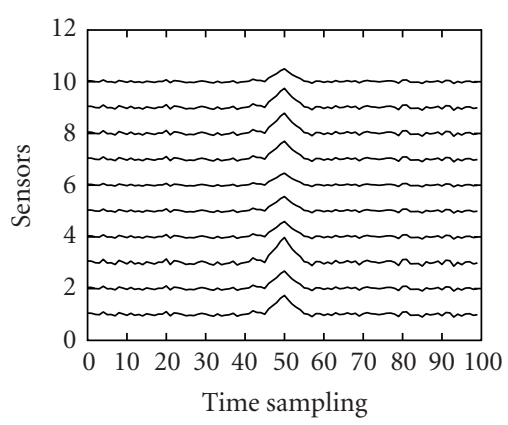

(g)

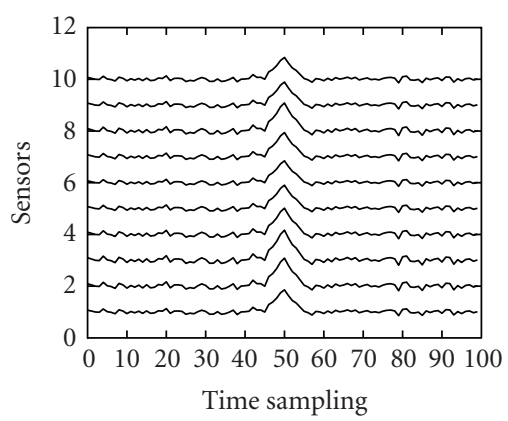

(j)

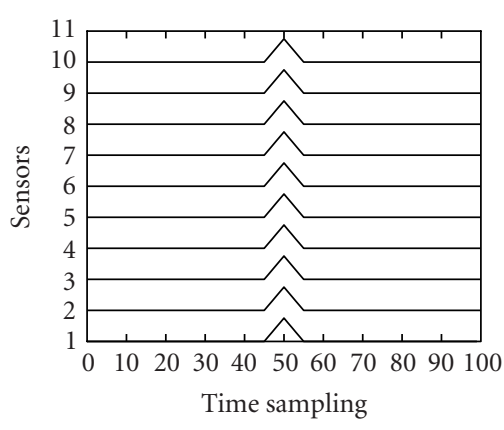

(b)

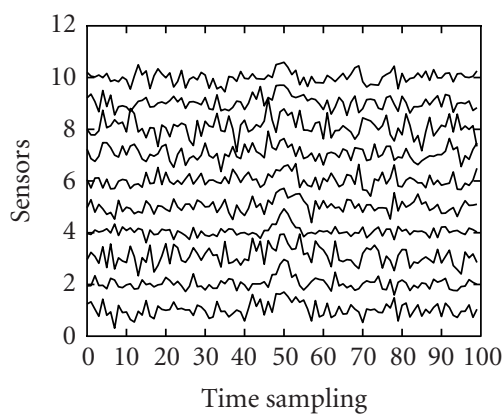

(e)

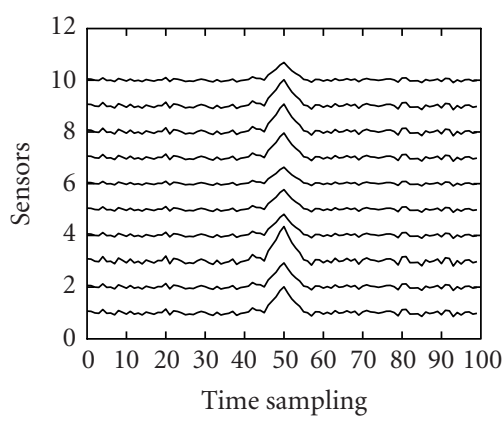

(h)

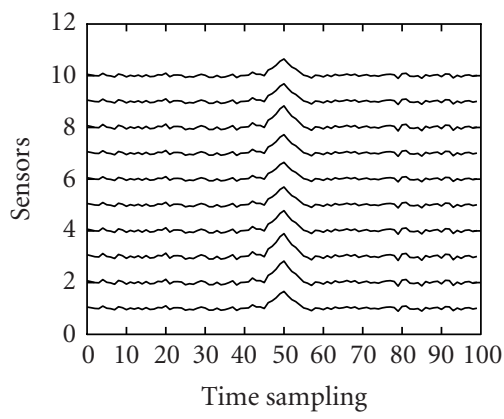

(k)

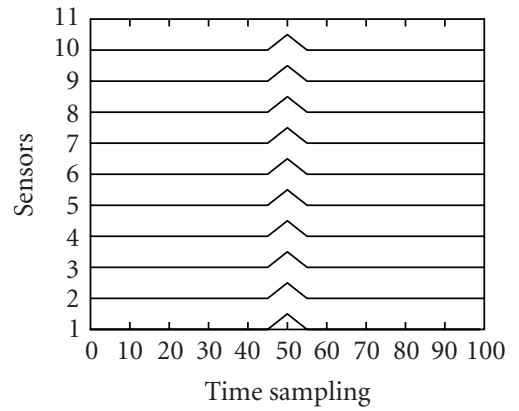

(c)

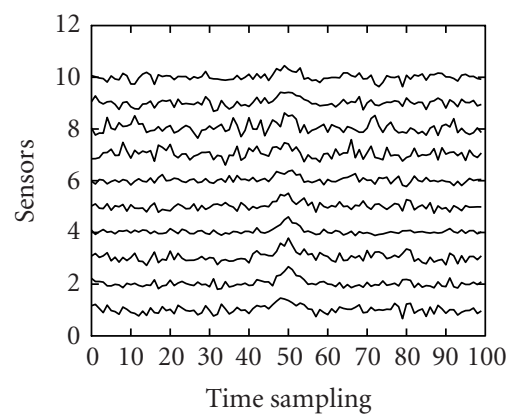

(f)

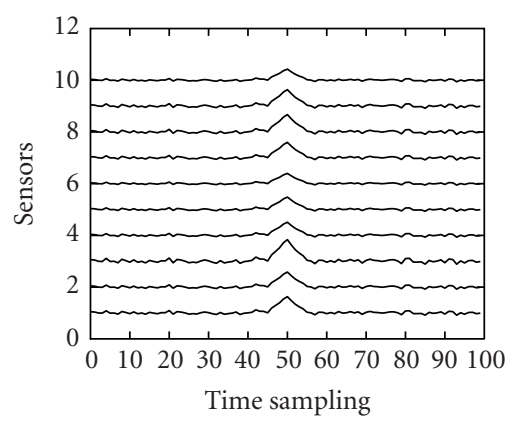

(i)

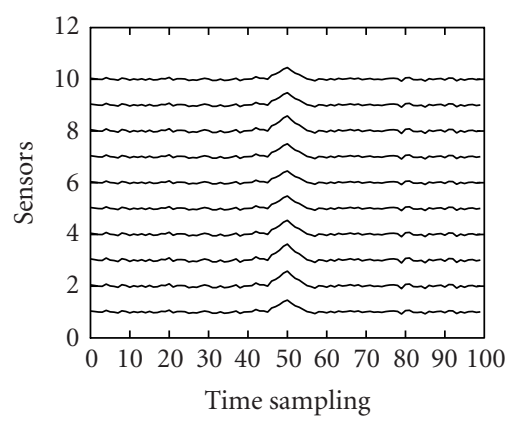

(1)

Figure 4: Comparison of (g), (h), (i) classical LRTA-(1, 1, 1)-based and (j), (k), (l) fourth-order-cumulant-slice-matrix-LRTA-(1, 1, 1)-based multiway filterings applied to a multicomponent seismic signal: (a) channel 1, (b) channel 2, and (c) channel 3, with (d), (e), (f) correlated Gaussian noise $(\mathrm{SNR}=-2 \mathrm{~dB})$. 


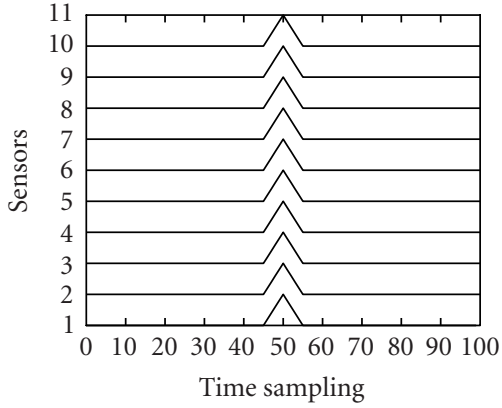

(a)

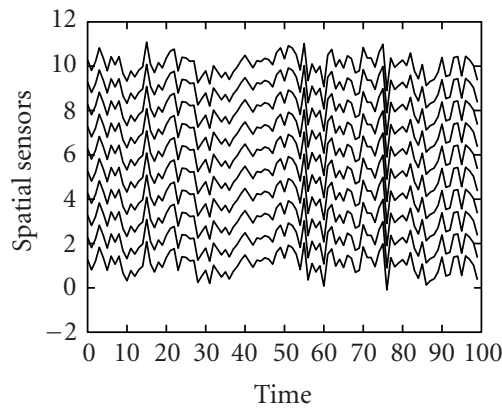

(d)

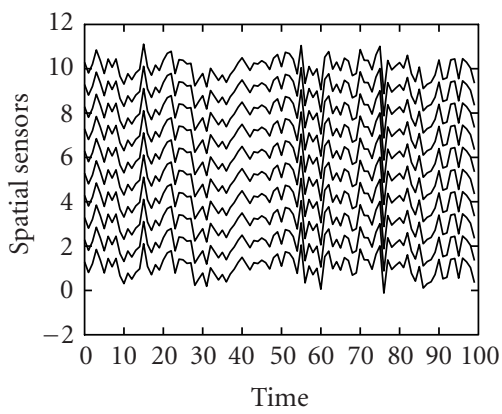

(g)

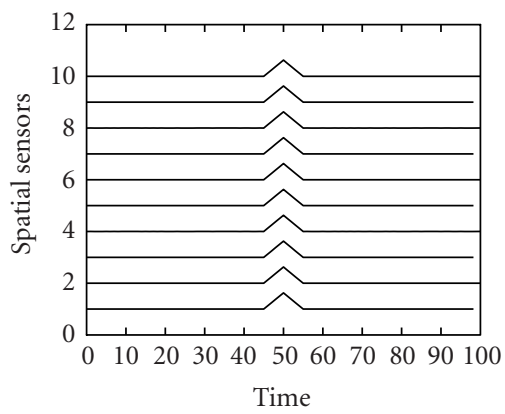

(j)

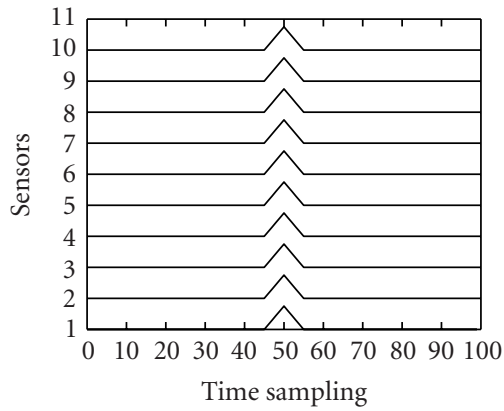

(b)

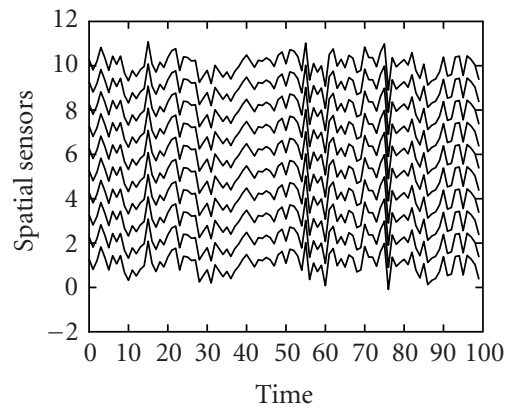

(e)

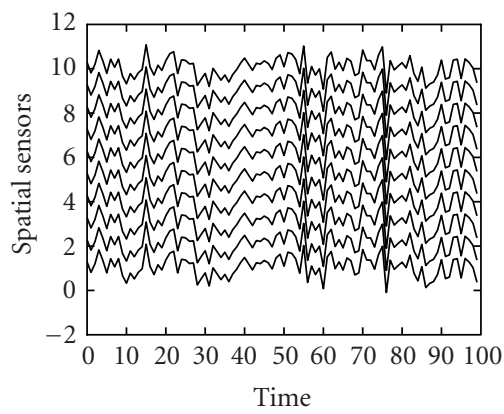

(h)

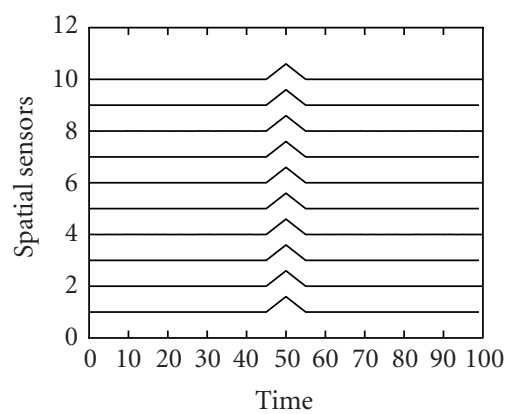

(k)

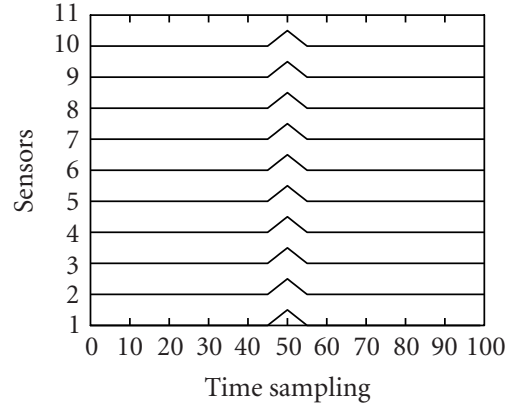

(c)

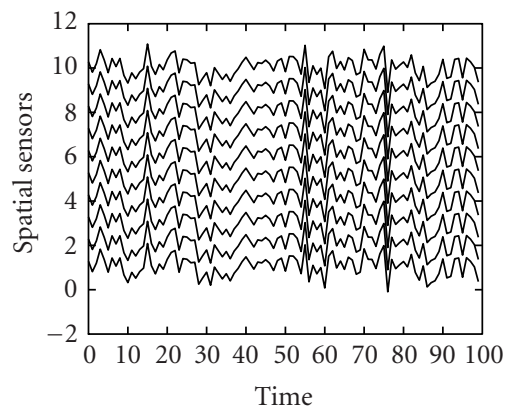

(f)

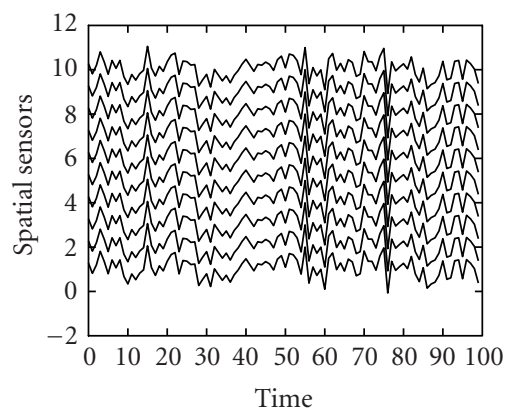

(i)

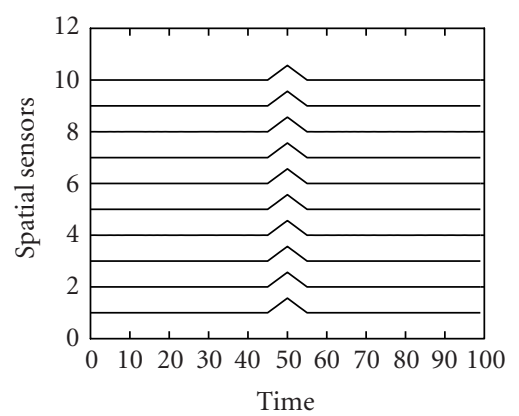

(1)

FIGURE 5: Comparison of classical LRTA-(1,1,1)-based and fourth-order-cumulant-slice-LRTA-(1, 1, 1)-based multiway filterings applied to a multicomponent seismic signal with totally correlated Gaussian noise (SNR $=-10 \mathrm{~dB})$. 


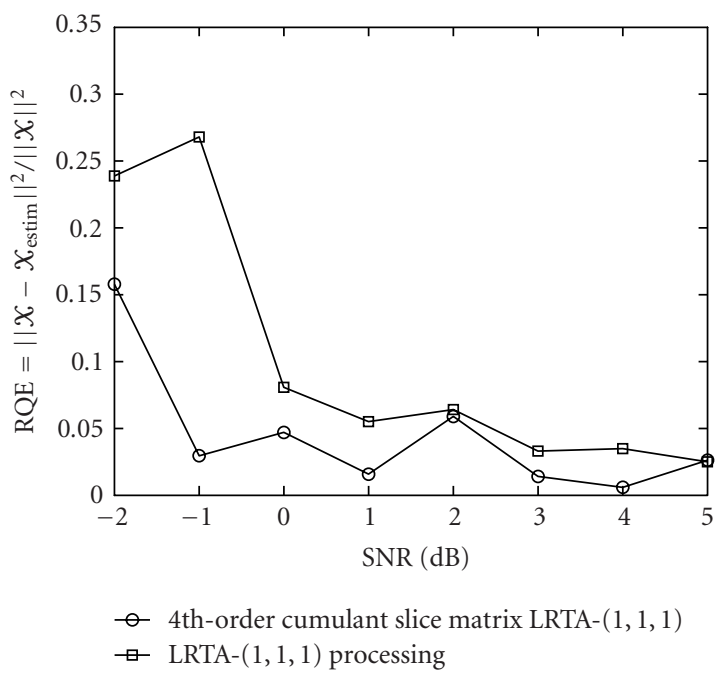

FIgURE 6: RQE evolution with respect to SNR (dB) for fourthorder-cumulant-slice-matrix-LRTA- $(1,1,1)$-based (circle) and classical LRTA-(1,1,1)-based (square) multiway filterings applied to a multicomponent seismic wave with correlated Gaussian noise (Figure 4).

On the contrary, the fourth-order-cumulant-slicematrix-LRTA- $(1,1,1)$-based multiway filtering of data tensor $\mathcal{R}$ is able to retrieve the initial signal tensor. The three components of the corresponding estimated signal $\widehat{X_{2}}$ are represented, respectively, on Figures 5j, 5k, and 51.

Finally, the evolution of the RQE with respect to the SNR, represented on Figure 2, shows that for the range of SNR going from $-10 \mathrm{~dB}$ to $10 \mathrm{~dB}$, the fourth-order-cumulant-slicematrix-LRTA- $(1,1,1)$-based multiway filtering gives better results than the classical LRTA- $(1,1,1)$-based multiway filtering since, all over the SNR range, the RQE given by the proposed method is lower than the one given by the classical method.

We also note that, like in Section 5.2.1 (Figure 1), Figure 2 also presents a comparison between the RQE obtained with the fourth-order-cumulant-slice-matrix-LRTA$(1,1,1)$-based (circle) and with the fourth-order-cumulanttensor LRTA- $(1,1,1)$-based (stars) multiway filtering. From these two curves, it appears, like in the color image processing case, that the multiway filtering of a multicomponent seismic signal based on the cumulant slice matrix and the one based on the corresponding fourth-order cumulant tensor give sensibly the same results.

\subsection{Further works}

Before concluding, we note that the optimal parameters $\left(K_{1}, K_{2}, K_{3}\right)$ determination is not the purpose of this paper and needs further investigations which would extend, for example, to the well-known array processing Akaike information criterion (AIC) or minimum description length (MDL) criteria $[39,40]$.

\section{CONCLUSION}

In this paper, an improvement of the multiway and multidimensional filtering of data tensor has been proposed.

The classical method is based on lower-rank- $\left(K_{1}, \ldots, K_{N}\right)$ tensor approximation which is commonly performed by the alternative least square process called TUCKALS3 algorithm. This algorithm determines simultaneously each $n$-mode projector on the $n$-mode signal subspace that is applied by means of the $n$-mode product operator on the $n$-mode of the data tensor. The $n$-mode projectors perform the projection on the eigenvectors associated with the largest eigenvalues of a matrix which can be assimilated as the covariance matrix of the data tensor $n$-mode vectors defined as the column vectors of the data tensor $n$-mode unfolding matrix.

The main idea of the proposed method is to replace the covariance matrix involved in TUCKALS3 algorithm with the fourth-order-cumulant-slice matrix of the data tensor $n$ mode vectors in order to remove the Gaussian components of an additive correlated Gaussian noise.

The good qualitative results of this new multiway filtering method are shown for noise reduction in a color image and multicomponent seismic wave in the case of an additive correlated Gaussian noise.

\section{ACKNOWLEDGMENT}

The authors would like to thank the anonymous reviewers for their careful reading and their helpful remarks, which have contributed to improving the clarity of the paper.

\section{REFERENCES}

[1] P. Comon, "Tensor decompositions," in Mathematics in Signal Processing V, J. G. McWhirter and I. K. Proudler, Eds., pp. 124, Clarendon Press, Oxford, UK, 2002.

[2] N. Le Bihan, Traitement algébrique des signaux vectoriels: Application à la séparation d'ondes sismiques, Ph.D. thesis, Institut National Polytechnique de Grenobl, Grenoble, France, 2001.

[3] D. Muti and S. Bourennane, "Multidimensional estimation based on a tensor decomposition," in Proc. IEEE Workshop on Statistical Signal Processing (SSP '03), pp. 98-101, St. Louis, Mo, USA, September-October 2003.

[4] D. Muti and S. Bourennane, "Multidimensional signal processing using lower-rank tensor approximation," in Proc. IEEE International Conference on Acoustics, Speech, and Signal Processing (ICASSP '03), vol. 3, pp. 457-460, Hong Kong, China, April 2003.

[5] D. Muti and S. Bourennane, "Traitement multidimensionnel du signal par décomposition tensorielle," in Proc. Colloque GRETSI sur le Traitement du Signal et des Images (GRETSI '03), Paris, France, September 2003.

[6] L. R. Tucker, "Some mathematical notes on three-mode factor analysis," Psychometrika, vol. 31, no. 3, pp. 279-311, 1966.

[7] P. M. Kroonenberg and J. De Leeuw, "Principal component analysis of three-mode data by means of alternating least squares algorithms," Psychometrika, vol. 45, no. 1, pp. 69-97, 1980.

[8] L. De Lathauwer, Signal processing based on multilinear algebra, Ph.D. thesis, Katholieke Universiteit Leuven, Department of Electrical Engineering, Leuven, Belgium, 1997. 
[9] L. De Lathauwer, B. De Moor, and J. Vandewalle, “On the best rank-1 and rank- $\left(R_{1}, R_{2}, \ldots, R_{N}\right)$ approximation of higher-order tensors," SIAM J. Matrix Anal. Appl., vol. 21, no. 4, pp. 1324-1342, 2000.

[10] G. H. Golub and C. F. Van Loan, Matrix Computations, The Johns Hopkins University Press, Baltimore, Md, USA, 3rd edition, 1996.

[11] J. B. Kruskal, "Rank, decomposition, and uniqueness for 3way and $\mathrm{N}$-way arrays," in Multiway Data Analysis (Rome, 1988), R. Coppi and S. Bolasco, Eds., pp. 7-18, North-Holland Publishing, Amsterdam, The Netherlands, 1989.

[12] J. B. Kruskal, "Three-way arrays: rank and uniqueness of trilinear decomposition, with application to arithmetic complexity and statistics," Linear Algebra and Appl., vol. 18, no. 2, pp. 95-138, 1977.

[13] R. A. Harshman and M. E. Lundy, "The PARAFAC model for three-way factor analysis andmultidimensional scaling," in Research Methods for Multimode Data Analysis, H. G. Law, C. W. Snyder Jr., J. A. Hattie, and R. P. McDonald, Eds., pp. 122-215, Praeger, New York, NY, USA, 1970.

[14] N. Sidiropoulos and R. Bro, "On the uniqueness of multilinear decomposition of $n$-way arrays," J. Chemom., vol. 14 , no. 3, pp. 229-239, 2000.

[15] R. Bro, Multi-way analysis in the food industry, Ph.D. thesis, Royal Veterinary and Agricultural University, Frederiksberg, Denmark, 1998.

[16] N. Sidiropoulos, R. Bro, and G. Giannakis, "Parallel factor analysis in sensor array processing," IEEE Trans. Signal Processing, vol. 48, no. 8, pp. 2377-2388, 2000.

[17] N. Sidiropoulos, G. Giannakis, and R. Bro, "Blind PARAFAC receivers for DS-CDMA systems," IEEE Trans. on Signal Processing, vol. 48, no. 3, pp. 810-823, 2000.

[18] E. Kofidis and P. A. Regalia, "On the best rank-1 approximation of higher-order supersymmetric tensors," SIAM J. Matrix Anal. Appl., vol. 23, no. 3, pp. 863-884, 2002.

[19] T. Zhang and G. H. Golub, "Rank-one approximation to high order tensors," SIAM J. Matrix Anal. Appl., vol. 23, no. 2, pp. 534-550, 2001.

[20] T. G. Kolda, “Orthogonal tensor decomposition," SIAM J. Matrix Anal. Appl., vol. 23, no. 1, pp. 243-255, 2001.

[21] M. A. O. Vasilescu and D. Terzopoulos, "Multilinear image analysis for facial recognition," in Proc. 16th IEEE International Conference on Pattern Recognition (ICPS' 2002), vol. 2, pp. 511-514, Quebec City, Canada, August 2002.

[22] H. Wang and N. Ahuja, "Facial expression decomposition," in Proc. 9th IEEE International Conference on Computer Vision (ICCV'03), vol. 2, pp. 958-965, Nice, France, October 2003.

[23] S. L. M. Freire and T. J. Ulrych, "Application of singular value decomposition to vertical seismic profiling," Geophysics, vol. 53, pp. 778-785, 1988.

[24] M. Hemon and D. Mace, "The use of Karhunen-Loeve transform in seismic data prospecting," Geophysical Prospecting, vol. 26, pp. 600-626, 1978.

[25] K. Hsu, "Wave separation and feature extraction of acoustic well-logging waveforms using karhunen-loeve transformation," Geophysics, vol. 55, no. 2, pp. 176-184, 1990.

[26] G. M. Jackson, I. M. Mason, and S. A. Greenhalgh, "Principal component transforms of triaxial recordings by singular value decomposition," Geophysics, vol. 56, no. 4, pp. 528-533, 1991.

[27] X. Liu, "Ground roll suppression using the Karhunen-Loeve transform," Geophysics, vol. 64, no. 2, pp. 564-566, 1991.

[28] J. Mars, Séparation d'ondes, Ph.D. thesis, Institut National Polytechnique de Grenobl, Grenoble, France, 1988.
[29] F. Glangeaud and J.-L. Mari, Wave Separation, Editions Technip, Paris, France, 1994.

[30] S. Bourennane and A. Bendjama, "Locating wide band acoustic sources using higher order statistics," Applied Acoustics, vol. 36, no. 3, pp. 235-251, 2002.

[31] N. Yuen and B. Friedlander, "Asymptotic performance analysis of blind signal copy using fourth-order cumulants," Internat. J. Adapt. Control Signal Process., vol. 48, pp. 239-265, 1996.

[32] N. Yuen and B. Friedlander, "DOA estimation in multipath: an approach using fourth-order cumulants," IEEE Trans. Signal Processing, vol. 45, no. 5, pp. 1253-1263, 1997.

[33] J. L. Lacoume, P. O. Amblard, and P. Comon, Statistiques d'ordre supérieur pour le traitement du signal, Masson, Paris, France, 1997.

[34] J. M. Mendel, “Tutorial on higher-order statistics (spectra) in signal processing and system theory: theoretical results and some applications," Proc. IEEE, vol. 79, no. 3, pp. 278-305, 1991.

[35] E. Gonen and J. M. Mendel, "Applications of cumulants to array processing. III. blind beamforming for coherent signals," IEEE Trans. Signal Processing, vol. 45, no. 9, pp. 2252-2264, 1997.

[36] C. Eckart and G. Young, "The approximation of a matrix by another of lower rank," Psychometrika, vol. 1, pp. 211-218, 1936.

[37] L. De Lathauwer, B. De Moor, and J. Vandewalle, "A multilinear singular value decomposition," SIAM J. Matrix Anal. Appl., vol. 21, no. 4, pp. 1253-1278, 2000.

[38] B. Porat and B. Friedlander, "Direction finding algorithms based on high-order statistics," IEEE Trans. Signal Processing, vol. 39, no. 9, pp. 2016-2024, 1991.

[39] H. Akaike, "A new look at the statistical model identification," IEEE Trans. Automat. Contr., vol. 19, no. 6, pp. 716-723, 1974.

[40] J. Rissanen, "A universal prior for integers and estimation by minimum description length," Annals of Statistics, vol. 11, no. 2, pp. 416-431, 1983.

Damien Muti graduated form the National Superior School of Physics of Marseille (ENSPM) in 2000. He received a research master (DEA) in optics, signal and image processing in 2001 from the same school. Since October 2001, he is working as a Ph.D. student in the Multidimensional Signal Processing Group (GSM), Fresnel Institute (CNRS UMR-6133), in Marseille, France. His research interest deals with ten-

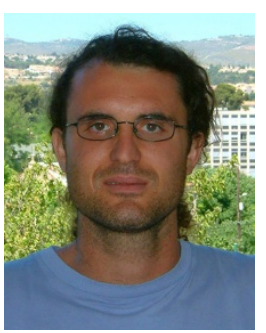
sor signal processing, array processing, filtering, with applications on multicomponent images, on polarized seismic signals, and other multicomponent signals that can be modelled by multiway arrays.

Salah Bourennane received his Ph.D. degree from Institut National Polytechnique de Grenoble, France, in 1990 in signal processing. Currently, he is a Professor at the École Généraliste d’Ingénieurs de Marseille, France. His research interests are in statistical signal processing, array processing, image processing, multidimensional signal processing, and performance analysis.

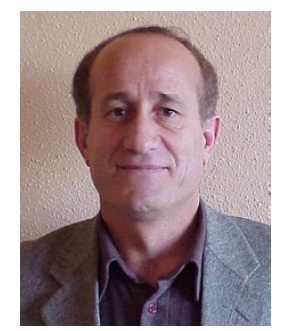

